\title{
Performance Characterization of NVMe-over-Fabrics Storage Disaggregation
}

\author{
ZVIKA GUZ, HARRY (HUAN) LI, ANAHITA SHAYESTEH, and VIJAY BALAKRISHNAN, \\ Samsung Semiconductor, Inc.
}

\begin{abstract}
Storage disaggregation separates compute and storage to different nodes to allow for independent resource scaling and, thus, better hardware resource utilization. While disaggregation of hard-drives storage is a common practice, NVMe-SSD (i.e., PCIe-based SSD) disaggregation is considered more challenging. This is because SSDs are significantly faster than hard drives, so the latency overheads (due to both network and $\mathrm{CPU}$ processing) as well as the extra compute cycles needed for the offloading stack become much more pronounced.

In this work, we characterize the overheads of NVMe-SSD disaggregation. We show that NVMe-overFabrics (NVMe-oF)-a recently released remote storage protocol specification-reduces the overheads of remote access to a bare minimum, thus greatly increasing the cost-efficiency of Flash disaggregation. Specifically, while recent work showed that SSD storage disaggregation via iSCSI degrades application-level throughput by $20 \%$, we report on negligible performance degradation with NVMe-oF-both when using stress-tests as well as with a more-realistic KV-store workload.
\end{abstract}

CCS Concepts: • Hardware $\rightarrow$ External storage; • Information systems $\rightarrow$ Flash memory; Storage management;

Additional Key Words and Phrases: NVMe-over-fabrics, performance characterization, network storage

\section{ACM Reference format:}

Zvika Guz, Harry (Huan) Li, Anahita Shayesteh, and Vijay Balakrishnan. 2018. Performance Characterization of NVMe-over-Fabrics Storage Disaggregation. ACM Trans. Storage 14, 4, Article 31 (December 2018), 18 pages. https://doi.org/10.1145/3239563

\section{INTRODUCTION}

NVMe-SDDs (i.e., PCle-based SSDs) are becoming increasingly popular for serving I/O intensive applications in data centers and enterprise deployments. This is because their vastly superior performance compared to SATA-SSDs and SAS-SSDs (in terms of both bandwidth and latency) caters well to the ever-increasing throughput demands of I/O intensive applications; primarily relational and NoSQL databases. A single current-generation NVMe-SSD sustains up to one million IOPS with a $90 \mu$ s read latency [56]-more than 5 times better than current generation SAS-SSDs, and

An earlier version of this article was published in Proceedings of the 10th ACM International Systems and Storage Conference (SYSTOR'17)

Authors' addresses: Z. Guz, H. (Huan) Li, A. Shayesteh, and V. Balakrishnan; Memory Platform Lab, Samsung Semiconductor, Inc., 3655 N 1st St, San Jose, CA 95134; emails: \{zvika.guz, harry.li, anahita.sh, vijay.bala@\}samsung.com.

Permission to make digital or hard copies of part or all of this work for personal or classroom use is granted without fee provided that copies are not made or distributed for profit or commercial advantage and that copies bear this notice and the full citation on the first page. Copyrights for third-party components of this work must be honored. For all other uses, contact the Owner/Author.

Copyright 2018 held by Owner/Author

1553-3077/2018/12-ART31

https://doi.org/10.1145/3239563 

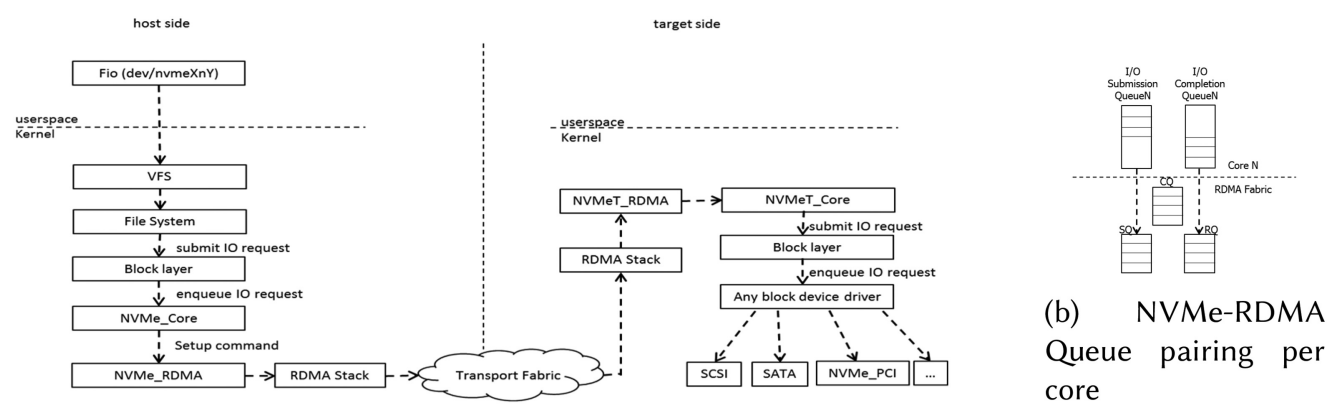

(a) NVMe-over-Fabrics Stack

Fig. 1. NVMe-over-Fabrics Architecture.

40 times better than SATA-SSDs. Indeed, many large-scale cloud companies have reported using PCIe-based SSDs as part of their infrastructure [19, 31, 49, 57].

Generally, storage devices can either be co-located within the compute server nodes, or be placed in dedicated storage nodes accessed through the network. Large-scale cloud companies originally used scale-out infrastructure, composing their data centers out of commodity servers that tightly coupled memory, storage, and compute [3, 38]. Unfortunately, this approach leads to inefficiencies and resource underutilization, because it fixes the ratio between compute, memory, storage, and network.

Resource underutilization in data centers is a common, well-documented phenomenon [3, 31, 36, 49]. Since the usage of different resources changes over time, predominantly independently from one another, there is no single static resource balance that fits every application that a server supports throughout its lifetime. Since changing these ratios at scale is economically unfeasible [51], server resources are often over-provisioned, leading to an increased total cost of ownership [3]. Specifically, NVMe-SSDs tend to be over-provisioned in terms of both load (IOPS) and capacity: capacity is over-provisioned to allow for future growth, and load is underutilized, because other software overheads tend to saturate the CPU well before reaching the drive limits [11, 49]. Indeed, PCIe-based Flash has been argued to be "problematically fast" [64].

Resource disaggregation is a common approach to mitigate the problem of over-provisioning. Specifically, storage disaggregation decouples compute and storage to different nodes (i.e., different servers), allowing independent scaling of each resource according to dynamic needs. It provides more flexibility when tuning the infrastructure to specific loads, because compute and storage can be configured for concrete demands rather than for average or maximum expected loads. In turn, it can greatly increase data center cost efficiency by reducing resource waste.

Despite its potential merits, storage disaggregation comes at a cost. Since storage devices are accessed over a network, interconnect latencies are added to every storage access, adversely affecting access times. Furthermore, the additional network processing and the overheads of the remote storage protocol itself tax the CPU on both the storage server and the compute node, further degrading application throughput compared to a direct-attached configuration. Hard-drive (HDD) disaggregation is common, because HDD's low performance (hundreds of IOPS and milliseconds latencies) makes network and processing overheads negligible [1, 22, 34, 41, 51, 62]. NVMe-SSD disaggregation, however, is considered significantly more challenging: since these drives are ordersof-magnitude faster than HDDs, the network latencies and protocol overheads become much more pronounced. A recent study on NVMe-SSD disaggregation via iSCSI demonstrated the cost benefits of Flash disaggregation but also reported an average degradation of $20 \%$ in application-level performance [31]. 
NVMe-over-Fabrics (NVMe-oF) [48] is a recent protocol standard for accessing NVMe devices over RDMA-capable networks (see Section 2). By leveraging RDMA, NVMe-oF offloads data movement to the network card (NIC), thus reducing the processing overheads involved in handling remote I/O requests on both the host and the target. Since processing overheads are a major cause for performance degradation when using disaggregated NVMe-SSD (see Section 4), NVMe-oF is expected to be a better fit as the underlining remote storage protocol for disaggregated storage. However, being a relatively new standard, there is little available performance characterization data of NVMe-oF and its overheads.

This article revisits NVMe-SSD disaggregation, using NVMe-oF as the remote storage protocol. We show that NVMe-oF minimizes the performance degradation experienced by the application, thus significantly improving the efficiency and viability of NVMe disaggregation. We also present a detailed NVMe-oF performance analysis. First, in Section 4, we use synthetic stress-tests to characterize and break down NVMe-oF latency and throughput overheads, and compare them to those of iSCSI. Then, in Section 5, we use RocksDB and MySQL as examples for real-world I/O intensive workloads, and show that they experience minimal performance degradation when running on remote storage. Furthermore, we show that NVMe-oF's low processing overhead enables better scaling at the storage server side compared to iSCSI, improving cost efficiency. Lastly, in Section 6, we show that the processing efficiency and scalability of the storage server can be further improved via the use of SPDK NVMe-oF Target [25] with no modifications to the host nor the applications.

In summary, this article argues that NVMe-oF facilitates NVMe-SSD disaggregation: It preserves all the advantages discussed in previous literature but further minimizes performance impacts and thus significantly improves cost efficiency.

\section{NVME-OVER-FABRICS}

Non-Volatile Memory Express (NVMe) is an optimized, high-performance, software interface standard for accessing local non-volatile memory devices over PCIe [20]. NVMe is based on a high number of deep, paired Submission and Completion Queues, allocated in host memory. These paired, parallel queues are the interface between the NVMe driver and the NVMe controller, which manage them cooperatively. While other proprietary protocols exist $[4,16], \mathrm{NVMe}$ is the predominate and most widespread technology for PCIe-based SSD devices. In this article, we use the terms PCIe-based SSD and NVMe-SSD interchangeably.

NVMe-over-Fabrics [48] is a recent extension to the NVMe standard that enables access to remote NVMe devices over different network fabrics. It eliminates unnecessary protocol translations (such as SCSI) along the I/O path to the remote device, exposing the multiple paired-queue design of NVMe. The goal is to provide applications with fast storage access regardless of whether NVMeSSDs are attached locally or accessed remotely; NVMe-oF aims to keep the overheads of remote access to less than $10 \mu \mathrm{s}$. NVMe-oF currently supports two types of fabric transport: RDMA and Fiber Channel. Of the two, RDMA enjoys wider availability both in traditional HPC domain as well as in modern data centers [8, 21, 30, 32, 37, 42, 61].

Remote Direct Memory Access (RDMA) is a remote memory management capability that allows direct data movement from one machine's memory to another machine's memory over the network without CPU involvement [52]. RDMA enables low latency, high-throughput remote access by (1) using zero-copy networking: data is transferred directly to or from application memory, eliminating the need to copy data between network layers as done in TCP/IP; (2) using kernel bypass: applications perform data transfers directly from user-space without kernel involvement; and (3) eliminating CPU involvement: RDMA transfers do not consume CPU cycles or pollute the CPU caches. 
RDMA can be implemented over different link layer protocols. It was originally associated with InfiniBand [35], requiring special-purpose interconnect. Two recent Ethernet-base alternativesRoCE and iWARP-provide a more cost-efficient solution that is expected to increase RDMA adoption in the data center domain. RoCE (RDMA over Converged Ethernet) [7] is based on InfiniBand transport over Ethernet and uses RDMA over Ethernet and UDP/IP frames. iWARP (interent Wide Area RDMA Protocol) [9] uses RDMA over a connection-oriented transport such as TCP. Both iWARP and RoCE require specialized RDMA-capable NICs, but can both operate over modern Ethernet-based switches. This greatly reduces the infrastructure investment compared to InfiniBand. ${ }^{1}$ While running iWARP over standard Ethernet is cheaper than running RoCE, RoCE provides lower latencies and has a strong following. Lately, RoCE seems to be gaining momentum and is being adopted by OEMs (e.g., Reference [47]); RoCEv2 is the fabric used in our NVMe-oF evaluation.

Figure 1(a) shows the stack of NVMe-over-Fabrics for the host and target. The Host driver contains common NVMe host core, and PCIe and RDMA transports. The Target driver contains NVMe target core, and RDMA transport. Both drivers share common NVMe and NVMe-oF data structures, queuing interfaces, commands, and properties, which are all independent of the NVMe Transport layer. NVMe Transport binding (NVMe-RDMA block), binds NVMe-over-Fabrics to NVMe Transport layer and is specific to the Fabric Protocol and physical layer.

Before a transfer is made, the host makes an association (connection) to the NVM controller in the target, creating a one-to-one mapping between I/O Submission Queues and I/O Completion Queues. NVMe queues are aligned to CPU cores, and paired with RDMA dedicated Send (SQ) and Response (RQ) Queue Pairs and Completion Queues (see Figure 1(b)). This queue design with endto-end pairing exposes the inherent parallelism of NVMe to the remote host. While the association exists, NVMe Host Driver encapsulates the NVMe commands and responses into a fabric-neutral Command Capsule and passes it to the NVMe RDMA Transport. A capsule is the NVMe unit transferred from the host to the NVM subsystem [48].

\section{METHODOLOGY}

This section describes the hardware and software setup used in our study. Our non-disaggregated baseline configuration uses a dual-socket Xeon E5-2690 v4 server [23] with 128GB DDR4 memory. It is fitted with a single PM1725 NVMe-SSDs [56]-a high-end enterprise drive that supports up to 750 (120) KIOPS of random read (write) traffic, and 3GB/s (2GB/s) of sequential read (write) bandwidth. We refer to this configuration as Direct-Attached-Storage (DAS), or local server. When evaluating remote storage, we use three such DAS servers and move their drives to a designated storage server (referred to as target). The three baseline nodes now access the drives over the network, and are referred to as hosts (or datastore servers [31]). The target node is a quad-socket Xeon E7-8890 v4 server [23], populated with 512GB DDR4 memory and the same three NVMe-SSD drives. To reduce noise and allow for a cleaner comparison, we disable hyperthreading as well as power-saving states on all servers.

All servers use a single ConnectX-4 100Gb Ethernet NIC [39] that provides RDMA over Ethernet (RoCE) capabilities, and connect through a $100 \mathrm{~Gb}$ Ethernet switch [40]. For the remote storage configuration, we evaluate both iSCSI and NVMe-oF over RoCE. Since data centers mainly use Ethernet, we use RoCE rather than InfiniBand for the underlying interconnect (as described in

\footnotetext{
$\overline{{ }^{1} \text { Unlike iWARP }}$, RoCE requires that switches support Data Center Bridging (DCB). While old switches do not support DCB, it is becoming common in new high-bandwidth switches.
} 
Section 2). For NVMe-oF, we set the number of I/O queues to 44 per remote drive. We made an explicit effort to tune the iSCSI setup, following known best practices (e.g., [31]).

We use two classes of workloads in our performance analysis. First, for stress-testing, we use fio [2] to generate synthetic $\mathrm{I} / \mathrm{O}$ traffic. We use $4 \mathrm{~K}$ random traffic to create the highest load on the CPUs and on the remote storage stacks. We also bypass the operating system page cache by using fio's direct I/O mode. Then, to simulate more-realistic data center workloads, we use RocksDB and MySQL as examples for two I/O-intensive applications that are widely used in many data centers production stacks $[14,46]$.

RocksDB [15] is a popular KV store library that is used both as an embeddable single-node $\mathrm{KV}$ database, and as a storage engine within larger distributed Database Management Systems (DBMS). We use RocksDB's own benchmark utility-db_bench-to generate loads and to measure performance. We model $16 \mathrm{~B}$ keys with $800 \mathrm{~B}$ and $10 \mathrm{~KB}$ values, and use a $80 / 20$ read-write mix. While some RocksDB installations might be used with larger objects [31], we chose smaller objects to further stress our system. We also limit the OS page cache so that more requests would be served from the drives rather than from DRAM.

MySQL [45] is an open-source Relational Database Management System (RDBMS). We use TPCC [10]-an online transaction procession (OLTP) workload-to characterize MySQL performance. Specifically, we use tpcc-mysql [33] to create the database, generate the load, and measure performance (reported in transactions-per-minute (tpmC)). We set the database size to 500 -warehouses (50GB), and run two independent MySQL instances on each datastore server with 150 connections per instance. In all configurations (DAS, NVMe-oF, and iSCSI), we use separate drives to store the database and MySQL log file, as it improves overall performance in all cases. Since our MySQL study focuses on evaluating Flash disaggregation scalability (see Section 5.2), we use up to ten datastores servers (rather than three as in the other experiments), and populate the target node with eight PM1725 drives (rather than three). We also use two additional clients to run tpcc-mysql and to drive the transactions. The clients' hardware is similar to that of the datastore servers, though their specification is unimportant for the sake of this study: In all cases, we verified that the clients are never the bottleneck. While all datastores run TPC-C, note that we simulate a multiple-database environment rather than a single clustered MySQL deployment. All instances are independent and each operates on its own copy of the database.

\section{NVME-OF PERFORMANCE ANALYSIS}

In this section, we use synthetic I/O traffic (generated with fio) to study the overheads of NVMeoF relative to a direct-attached storage. We also characterize remote storage access via iSCSI to quantify the performance savings realized through using NVMe-oF and RDMA. In Section 4.1 we examine performance at maximum load, and in Section 4.2, we characterize latencies and overheads at different system operation points.

\subsection{Maximum Load Characterization}

Figure 2 shows the maximum IOPS attained with different read-to-write ratios for DAS, iSCSI, and NVMe-oF. We use 16 jobs with 32 in-flight requests for DAS and NVMe-oF; and 44 jobs with 44 in-flight requests for iSCSI, as these configurations delivered the best performance for each setup. DAS saturates the three drives in all cases and serves as the upper limit for performance. As can be seen in the figure, NVMe-oF performance (red bars) is practically the same as DAS-the difference is less than $1 \%$ for all cases. The iSCSI throughput (green bars), however, is significantly lower than DAS and NVMe-oF: In the read-only test, iSCSI delivers 1.4 MIOPS, utilizing only $60 \%$ of the drives' available IOPS. iSCSI performance is hindered by an inefficient network stack and protocol processing overheads such as unnecessary protocol translation from NVMe to SCSI and back. In 


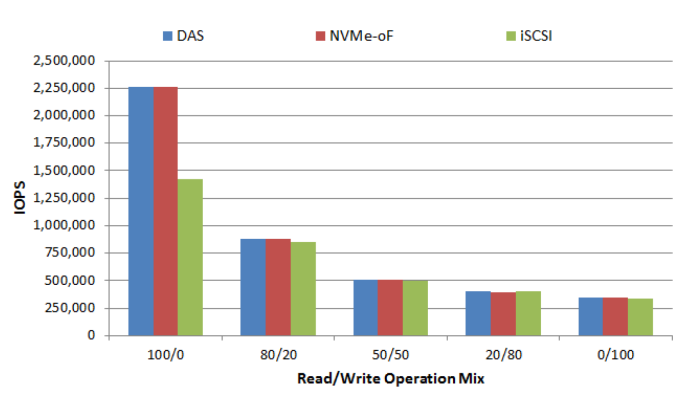

Fig. 2. Maximum achievable throughput (in IOPS) for DAS, NVMe-oF, and iSCSI.

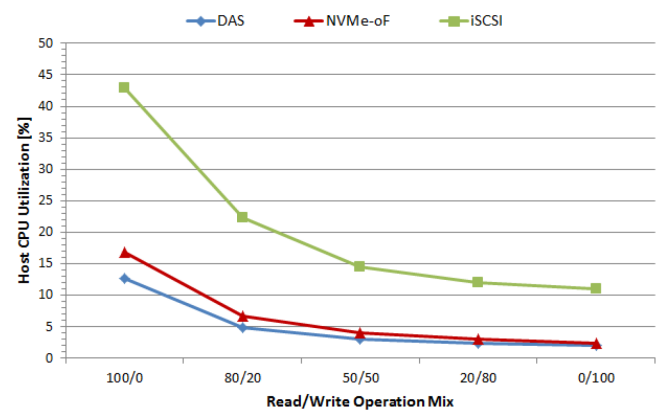

Fig. 3. Host CPU utilization for DAS, NVMe-oF, and iSCSI.

addition, and unlike NVMe-oF, iSCSI cannot expose NVMe's inherent parallelism and multiple queues, leaving significant performance on the table.

As more writes are added to the instruction mix, overall performance in all configurations decreases due to the drive's asymmetric read/write performance (One PM1725 drive can sustain 750 and 120 KIOPS for read and write traffic, respectively). When total IOPS, and thus the overall system load drop, iSCSI is able to support the reduced IOPS, achieving performance close to DAS and NVMe-oF. Note that in addition to inferior performance, iSCSI adds significant CPU overhead as shown in Figure 3. While NVMe-oF adds at most $4.3 \%$ to the overall host CPU utilization, iSCSI overheads can add a whopping $30 \%$. For real-world workloads that require more compute resources than fio, these overheads will further degrade the application performance as they significantly reduce the overall available system resources.

Figure 4 provides a more in-depth look at CPU utilization for the read-only test, showing percore utilization on both the host and the target. User time in Figure 4(a) indicates time spent running the fio application, where Sys time accounts for network and storage protocols, and for Kernel mode processing. In addition to system and user, the host in our iSCSI configuration spends significant time servicing software interrupts (soft). This is a known issue related to TCP/IP significant interrupt handling overheads; it can benefit from distributing interrupts via affinity setting [31]. In our experiments, we did not see noticeable improvements by setting interrupt affinity, probably due to the high core count of our machines. For the NVMe-oF host, overall CPU utilization is slightly increased from $13 \%$ in DAS to $17 \%$ in NVMe-oF, and processing is limited to one socket. Conversely, in the iSCSI setup, cores on both sockets in the host have a $43 \%$ average utilization.

On the target side, Figure 4(b) shows the utilization of the active cores in the system. Out of the 96 cores, only 24 cores are utilized, i.e., only one out of the four available sockets. The average utilization numbers reported in the figure account for all cores, which explains the relatively 


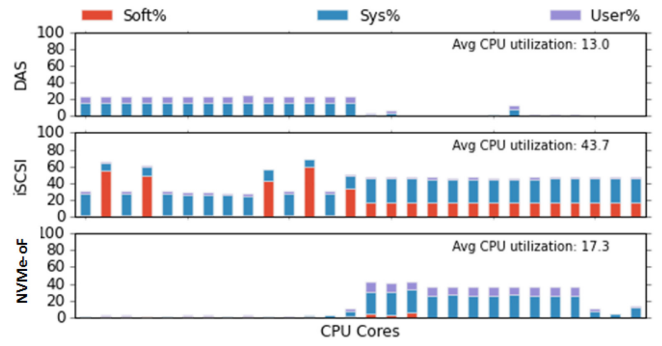

(a) Host

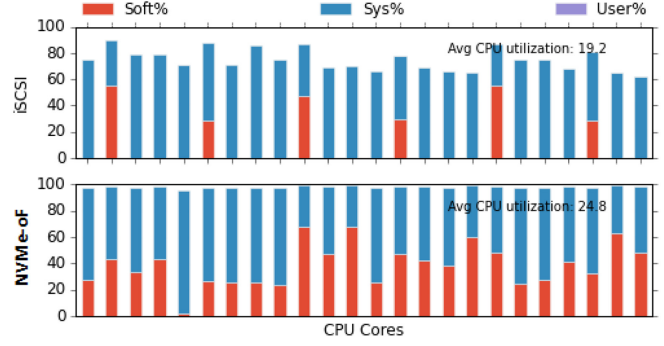

(b) Target

Fig. 4. Average per-core CPU utilization for both (a) the host and (b) the target during fio read-only test. We consider DAS as a host and show its data in (a). For the sake of presentation clarity, we only show the active cores on the target node.

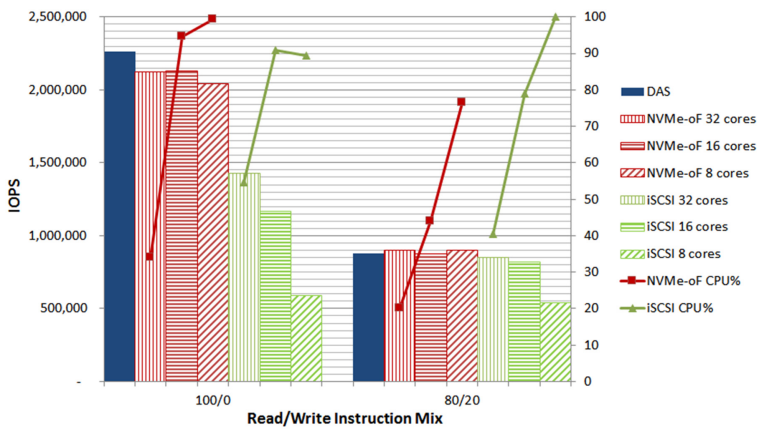

Fig. 5. Overall IOPS and cores utilization for different core counts on the target.

low numbers. Both NVMe-oF and iSCSI have high utilization on active cores. However, note that iSCSI's overall IOPS performance is $37 \%$ lower than NVMe-oF performance for the read-only test (see Figure 2).

Lastly, to further study the target overheads and compute requirements, we repeated the cpuintensive tests (read-only and 80/20 read/write) while enabling only a subset of the cores available on the the target. As can be seen in Figure 5, since NVMe-oF requires less CPU resources per IO, its performance degrades gracefully as the core count decreases. Even with only $1 / 12$ of the cores enabled (red diagonal stripes) NVMe-oF performance is still within $90 \%$ of local-storage for the read-only test. iSCSI performance degrades much more quickly as the number of available cores decreases. For the read-only case, performance falls by more than $2 \times$, to $26 \%$ of the max IOPS in the same scenario (green diagonal stripes). NVMe-oF's low overheads on the target node is particularly important for data center cost efficiency, because it allows storage servers to be populated with more drives and fewer processors (or lower-performance processors), thus reducing overall TCO without sacrificing performance.

\subsection{Latency and Throughput Analysis}

Figure 6 shows the latencies of DAS, NVMe-oF, and iSCSI for different request loads. We use a $4 \mathrm{~KB}$, read-only request stream, and show both average as well as tail (95th percentile) latencies. As can be seen in the figure, NVMe-oF performance is almost identical to DAS over the entire load range-the average latencies are within a 3.5\% difference except for the first (2\% load) run. In fact, NVMe-oF tail latencies are slightly better than DAS for light to medium-high loads. The same 


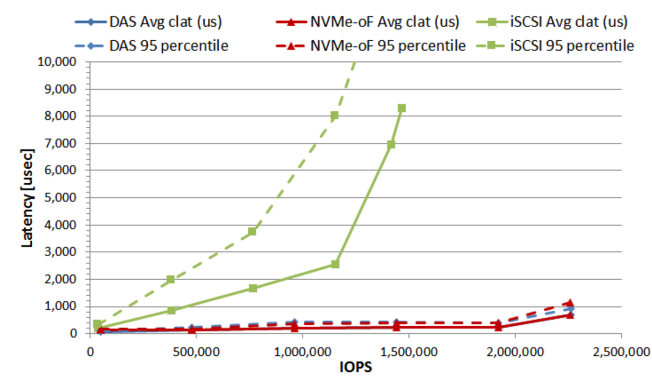

(a) Full view

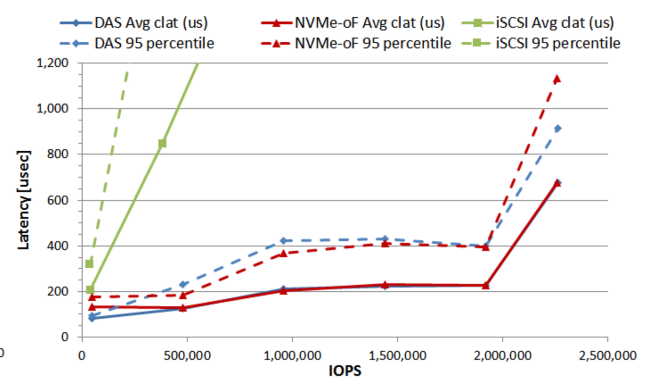

(b) Zoom-in on 0 -1ms range

Fig. 6. Average and tail latencies of DAS, NVMe-oF, and iSCSI for different request loads. Since iSCSI saturates early and exhibits significantly higher latencies, we show both the full range view (a), and a zoom-in on DAS and NVMe-oF (b).

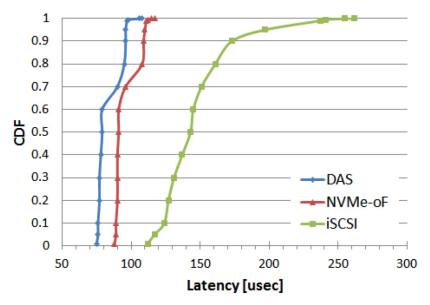

(a) Unloaded system

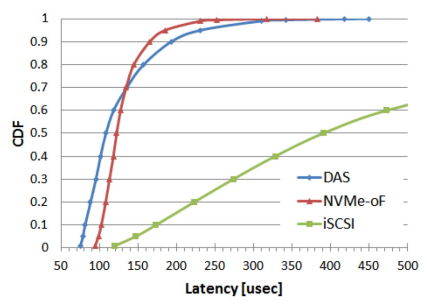

(b) $\mathrm{Load}=20 \%$ (480 KIOPS)

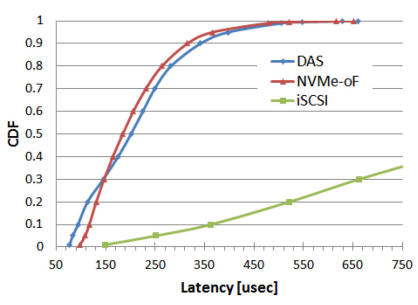

(c) $\mathrm{Load}=42 \%$ (960 KIOPS)

Fig. 7. Read latency CDF under different loads for DAS, NVMe-oF, and iSCSI.

phenomenon of slightly higher performance of NVMe-oF compared to DAS was also reported by Mintrum [43], and attributed to interrupt coalescing in the RDMA NIC. Indeed, under all practical loads scenarios, the performance of a locally attached NVMe-SSD and the performance of a remote NVMe-oF access are virtually the same.

Unlike NVMe-oF, iSCSI latencies are noticeably longer compared to local storage access. First, iSCSI saturates well before DAS (and NVMe-oF)-latencies start climbing exponentially after about 1.5 MIOPS compared to 2 MIOPS for DAS. Second, even for relatively light loads, iSCSI adds significant delays compared to the time needed to access local storage. When operating at $50 \%$ of drive capacity, the average latency is $2.5 \mathrm{~ms}-$ more than $10 \times$ the local access latency. The difference in tail latency is even larger: to keep the 95th percentile latency below $2 \mathrm{~ms}$, we can only operate at $17 \%$ of the maximum drive IOPS capacity. Conversely, the 95th percentile latency for DAS and NVMe-oF is kept below $2 \mathrm{~ms}$ throughout the entire load range. Our results indicate that, unlike iSCSI, NVMe-oF does not introduce additional latency to remote access, and can be deployed in even the most latency-sensitive applications without performance penalty.

To provide further comparison of NVMe-oF and DAS latencies, Figure 7 shows the cumulative distribution (CDF) of the end-to-end read latency under three different loads. The unloaded latencies (Figure 7(a); 1 job, 1 in-flight request) for all three configurations are relatively close: the average latencies are $83.5 \mu \mathrm{s}, 95.2 \mu \mathrm{s}$, and $145.8 \mu \mathrm{s}$ for DAS, NVMe-oF, and iSCSI, respectively. NVMe-oF adds $11.7 \mu$ s delay-very close to the specification's target of $10 \mu \mathrm{s}$. iSCSI adds $62 \mu \mathrm{s}$. While larger, this is still acceptable for most data center use cases. Similarly, tail latencies are also close: 95th percentile latencies are $96 \mu \mathrm{s}, 110 \mu \mathrm{s}$, and $197 \mu \mathrm{s}$ for DAS, NVMe-oF and iSCSI, respectively (i.e., $14 \mu$ s overhead for NVMe-oF and $101 \mu$ s overhead for iSCSI). However, as we increase the load on 


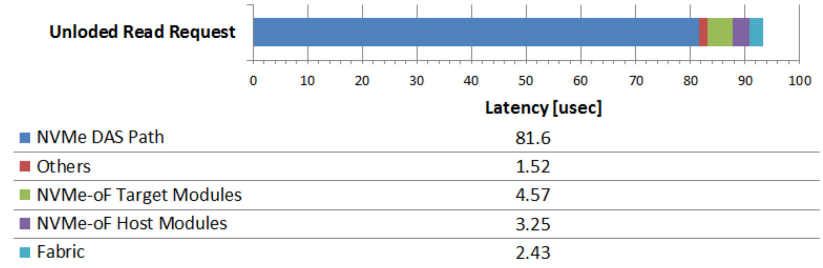

Fig. 8. A breakdown of NVMe-oF end-to-end latency for an unloaded read request.

the system (Figures 7(b) and 7(c)), iSCSI's latencies become much higher than DAS while NVMe-oF overheads (both average and tail) stay minimal. Since we wanted to focus on NVMe-oF in these figures, we let iSCSI go out of the figure range.

Lastly, Figure 8 shows a breakdown of unloaded NVMe-oF read latency. We use ftrace [54] to trace the time spent in each module, and we use a DAS read test to derive the baseline time needed for local access. Overall, NVMe-oF is $11.7 \mu$ s slower than a local NVMe access. The NVMeoF-related modules on the host account for $27 \%$ of the overhead (block layer, NVMe driver, and the RDMA stack; see Figure 1(a)); NVMe-oF target modules (RDMA stack and NVMe_T modules in Figure 1(a)) account for an additional 38\%. The network and switch add another $2.43 \mu$ s to the overall latency. Finally, an extra $13 \%$ is spent outside of any NVMe-oF module. While we do not have further breakdown of this latency, it includes interrupt delivery latency, kernel scheduling delays, and overhead related to switching between user and kernel spaces. Intel's SPDK [24]-the user-mode NVMe-oF driver-can further reduce these overheads, because SPDK runs in userspace (thus eliminating user-to-kernel transitions) and uses polling rather than interrupts (thus eliminating interrupt handling latencies) $[63,66]$. Indeed, our experiments running SPDK for DAS and for the NVMe-oF target node resulted in a reduction of $2.84 \mu \mathrm{s}$ in both configurations. In other words, using NVMe-oF SPDK Target [25] reduces NVMe-oF additional latency for unloaded read to $8.9 \mu$ s compared to DAS, and preserves the $11.7 \mu$ s overhead compared to local access with SPDK.

\section{DISAGGREGATED DATABASE AND KV-STORE}

While in Section 4 we use microbenchmark stress-tests to characterize NVMe-oF performance, real-world data center workloads can have very different system characteristics and are generally less demanding of a node's I/O system. Often, attached-drives are only moderately utilized [31, 49, 65], because other system bottlenecks saturate well before the drives. Moreover, compute-per-I/O is higher relative to our fio test, because (1) fio is inherently less compute-intensive than a fullfledged application, (2) DRAM caching filters out some of the I/Os, an effect not modeled by our direct fio test; and (3) various software overheads associated with data center services (coined "data center tax" by Kanev et al. [27]) further increase the processor load. In this section, we use RocksDB and MySQL-both widely used in many data centers production stacks [14, 46]-to characterize a more realistic Flash disaggregation scenario and to quantify its expected benefits.

\subsection{Disaggregated KV-Store}

Figure 9 shows RocksDB performance (in terms of operations-per-second) using local storage vs. remote storage via both iSCSI and NVMe-oF (See Section 3 for RocksDB and db_bench configuration details). We use $10 \mathrm{~KB}$ objects as our baseline, similar to Klimovic et al. [31], to represent typical RocksDB production loads. In addition, we also show performance with smaller $800 \mathrm{~B}$ objects. With smaller objects, more transactions are executed per second, further stressing the remote storage setup. As can be seen in the figure, while iSCSI performance is significantly lower than local storage ( $40 \%$ performance degradation), NVMe-oF performance is within a negligible $2 \%$ of 


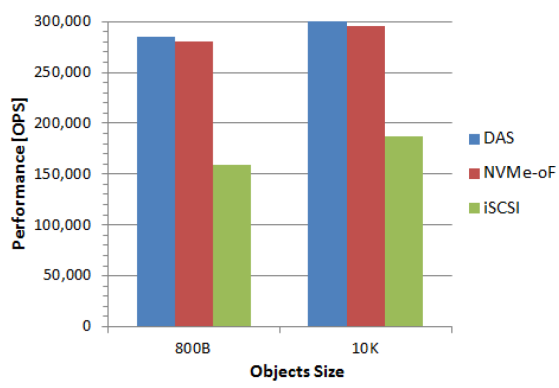

Fig. 9. RocksDB maximum throughput for DAS, NVMe-oF, and iSCSI. We show results for two object sizes: $800 \mathrm{~B}$ and $10 \mathrm{~KB}$.

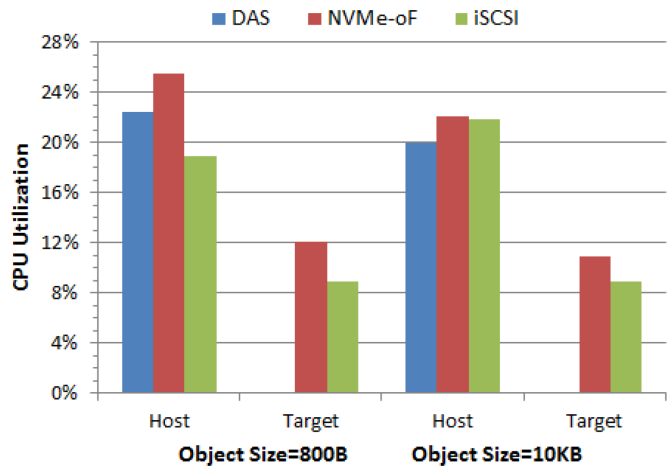

Fig. 10. Average CPU utilization on the host and target for RocksDB workloads.

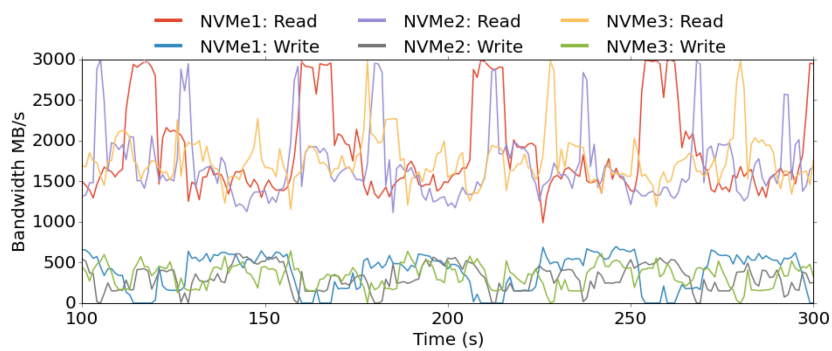

Fig. 11. Transient NVMe-SSD bandwidth on the NVMe-oF target node during 200s of RocksDB NVMe-oF run. All three drives gets saturated (3GB/s read) during the bandwidth spikes.

DAS. Indeed, in terms of overall performance, the host does not see much difference when the drives are accessed locally vs. remotely over NVMe-oF. In terms of processing overheads (see Figure 10), while NVMe-oF does increases the host CPU utilization by $10 \%$, this modest overhead should be acceptable in most scenarios. While the CPU utilization on the target is already relatively low, in Section 6, we show that it can be drastically decreased via the use of SPDK NVMe-oF target. The CPU utilization for iSCSI is given for completeness, but comparing it to NVMe-oF is difficult, because the overall performance is significantly lower.

Figure 11 shows the fluctuations in NVMe-SSD bandwidth on the NVMe-oF target node during the workload run. These bandwidth fluctuations are a property of RocksDB background processes and are an expected RocksDB behavior. As can be seen in the figure, the NVMe-oF target machine 


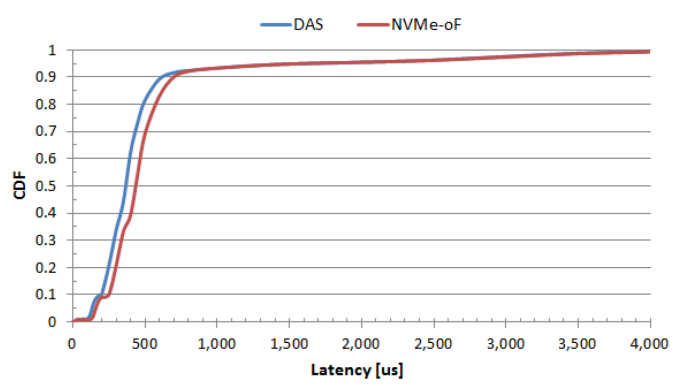

Fig. 12. RocksDB read latency CDF for DAS and NVMe-oF, with 800B objects.

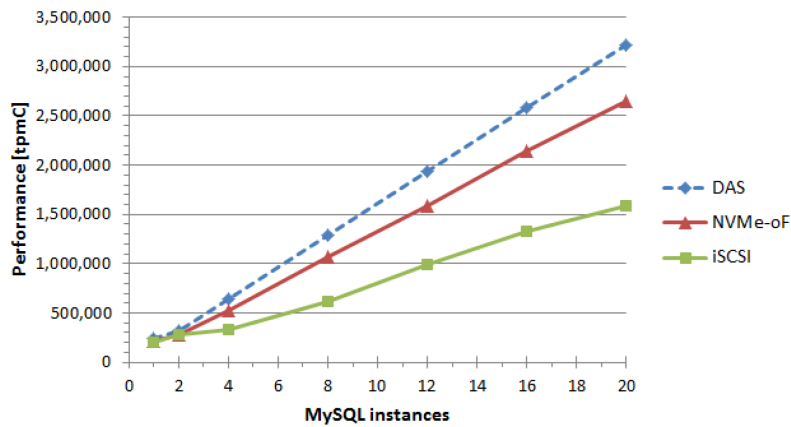

Fig. 13. TPC-C performance for DAS, NVMe-oF, and iSCSI, as the number of MySQL (and TPC-C) instances increases. Since the DAS configuration does not share resources across nodes, its scaling performance is projected from the performance of a single server.

is able to saturate all three NVMe drives: all drives reach $3 \mathrm{~GB} / \mathrm{s}$ (the drives peak read bandwidth) during periods of bandwidth spikes. Both DAS and NVMe-oF setups saturate the NVMe drives. However, note that in the DAS configuration each of the three hosts contain a single NVMe SSD, while for the NVMe-oF configuration the target machine handles all three drives with peak SSD bandwidth of $7.8 \mathrm{~GB} / \mathrm{s}$.

Lastly, Figure 12 plots the cumulative read latency distribution reported by db_bench for NVMeoF and DAS. For Figure 12, we use 800B objects and run at a reduced load (70\%), so that we do not operate close to saturation point. We omit iSCSI from this figure. Since we could not reach the same throughput when using iSCSI, any latency comparison would have been meaningless. NVMe-oF read latencies under load are comparable to local storage: NVMe-oF average latency is $11 \%(60 \mu \mathrm{s})$ higher than DAS, and the 99th percentile latency overhead is a negligible $2 \%(83 \mu \mathrm{s})$.

\subsection{Disaggregated RDBMS}

Figure 13 shows TPC-C performance (in terms of transactions-per-minute) using local storage vs. remote storage via both iSCSI and NVMe-oF as the number of MySQL instances is scaled up (See Section 3 for MySQL and TPC-C configuration details). As can be seen in the figure, while iSCSI performance trails behind as the number of servers increases, NVMe-oF performance scales well. With 20 MySQL instances, NVMe-oF performance is within $82 \%$ of DAS projected performance, whereas iSCSI performance is at a mere $49.2 \%$.

TPC-C is CPU intensive: The CPU utilization of the DAS server when running two MySQL instances is $86 \%$. At the same time, the NVMe-SSD is left underutilized: the total I/O bandwidth is only $612 \mathrm{MB} / \mathrm{s}-26 \%$ of the device's $3 \mathrm{~GB} / \mathrm{s}$ maximum bandwidth. Thus, storage disaggregation 


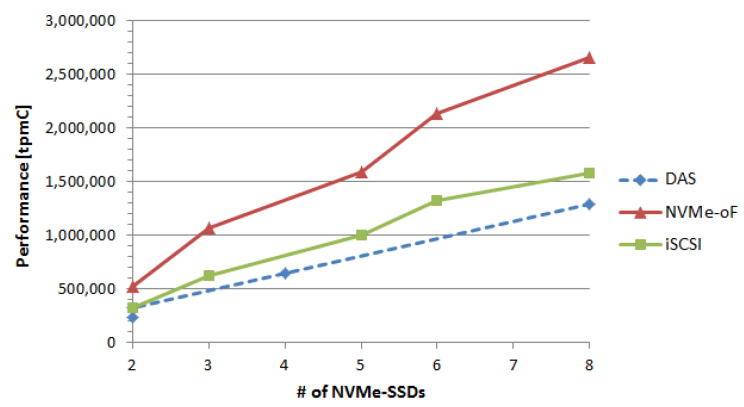

Fig. 14. Max TPC-C performance for DAS, NVMe-oF, and iSCSI as we increase the number of NVMe-SSD drives. Storage disaggregation allows sharing the drives across multiple MySQL servers, and delivers higher overall performance per drive. Unlike iSCSI, NVMe-oF performance is not hampered by protocol overheads and outperforms the local-storage configuration by more than $2 \times$.

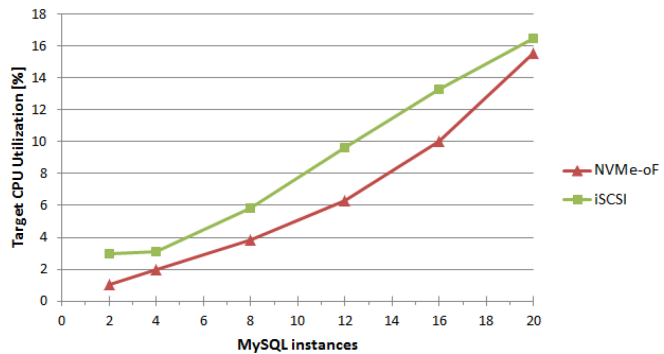

Fig. 15. Average CPU utilization on the target node when running an increasing number of TPC-C instances. While the CPU utilization of both configurations is similar, note that the overall performance of NVMe-oF is higher.

allows for a much more efficient resource utilization in this case as it allows the same drives to be shared across multiple MySQL instances. Indeed, when running 20 MySQL instances, the target node uses only eight drives whereas the local storage configuration would have used twenty. Figure 14 further shows this by plotting the maximum TPC-C performance for a given number of drives out of all the runs in Figure 13 for each of the three configurations. As can be seen in the figure, disaggregated storage delivers higher performance than DAS for the same number of drives, because the drives are shared across multiple MySQL instances; since the same number of drives supports higher number of MySQL instances, the overall TPC-C performance is higher. However, while iSCSI overheads limit performance, NVMe-oF delivers more than $2 \times$ the performance of DAS per drive.

Lastly, Figure 15 shows the CPU utilization of the target node for both iSCSI and NVMe-oF. As can be seen in the figure, the target CPU is lightly utilized and could have supported more datastore servers and more drives before ever becoming the bottleneck. We did not push scaling beyond 20 MySQL instances, because we exhausted the number of datastore servers we had available for our experiments.

\section{IMPROVING STORAGE SERVER EFFICIENCY WITH SPDK}

In a disaggregated storage environment, a dedicated storage server serves multiple compute nodes and is optimized for high storage capacity and high I/O performance. As shown is Section 4.1, NVMe-oF's low overheads on the target node enable storage servers to be populated with more 


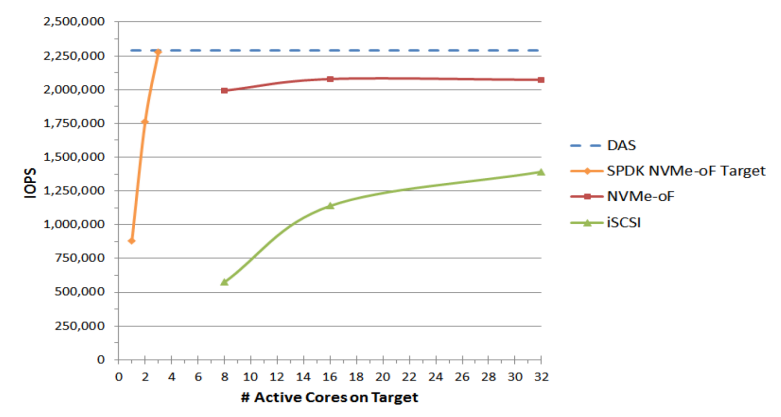

Fig. 16. Overall IOPS for different core counts on the target, for a $4 \mathrm{~KB}$ random-read fio workload. The blue dashed line shows the max IOPS in the system, measured by running in DAS mode.

drives and fewer processors, reducing overall TCO without sacrificing performance. In this section, we show that using SPDK-the user-mode NVMe-oF driver-data centers can further reduce the overheads on the target node and improve its efficiency without changing neither the compute node nor the applications.

The SPDK NVMe driver delivers higher performance than the kernel-based driver and is suitable for high-performance storage applications [63]. Specifically, by moving the driver to userspace, SPDK eliminates context switches and multiple data copies along the I/O path, thus reducing processing overhead and improving CPU utilization. While performance is better than that of the OS driver, a userspace driver is not a drop-in replacement, because applications need to be modified to explicitly manage the drives. In addition to the SPDK local driver, the SPDK toolkit contains an SPDK NVMe-oF target library [26] that provides all the primitives needed to run the entire NVMeoF target path in userspace. The toolkit also contains an SPDK NVMe-oF target application [25] that uses these primitives to implement a full userspace NVMe-oF target. Unlike the SPDK driver, running the SPDK NVMe-oF target application on the storage node does not require any changes on the host side. In this section, we run the SPDK NVMe-oF target application on the storage node and characterize the performance advantages and the reduction in CPU load.

Figure 16 shows the overall performance for a read-only fio workload, when only a subset of the 96 cores on the target are activated (for NVMe-oF and iSCSI, we set the number of active cores in the BIOS; for SPDK, we define the number of cores through a core mask when we enable SPDK target). The data for DAS, NVMe-oF, and iSCSI is the same as in Figure 5 and is provided here for ease of comparison. As can be seen in the figure, SPDK NVMe-oF target saturates all three NVMeSSDs using only three active cores. In contrast, NVMe-oF requires 16 cores to reach $90 \%$ of the max performance. Figure 17 further shows the CPU utilization of the active cores and the overall system utilization on the target during the same run. Unlike NVMe-oF and iSCSI, the utilization of the active cores when running with SPDK (the orange solid line) is always $100 \%$, because the driver runs in polling-mode. Overall system utilization however, is significantly lower with SPDK: with three cores, the target utilization is only $3.1 \%$ compared to $15.8 \%$ for NVMe-oF when running with 16 cores. This reduction in CPU load is important, since it facilitates scaling-a dedicated storage server can support a large number of devices and deliver very high I/O performance without the CPU becoming the bottleneck.

For completeness, Figure 18 shows the per-core CPU utilization on the target for SPDK when running on three cores. In contrast with NVMe-oF and iSCSI (see Figure 4(b)), the three active cores are fully utilized and stay solely in userspace.

Lastly, we rerun RocksDB with SPDK to exemplify the expected savings on the target when running a real-world data center workload. Figure 19 shows RocksDB performance (in terms 


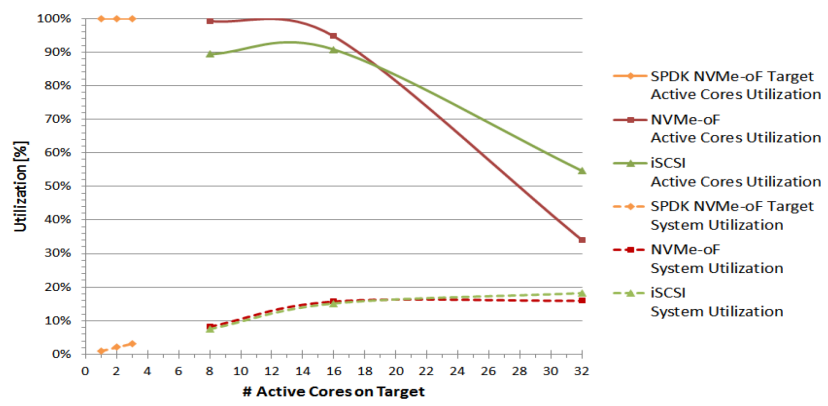

Fig. 17. System utilization (dashed lines) and active cores utilization (solid lines) on the target node for a $4 \mathrm{~KB}$ random-read fio workload.

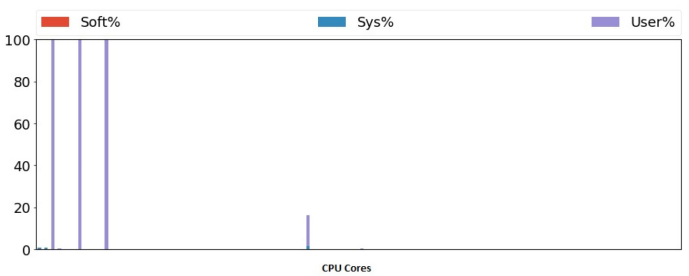

Fig. 18. Average per-core CPU utilization for the target node when running a 4KB random-read fio workload with SPDK NVMe-oF target on 3 cores. The cores stay solely in userspace.

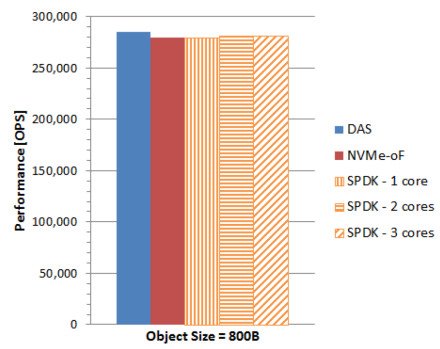

Fig. 19. RocksDB throughput for DAS, NVMe-oF, and SPDK NVMe-oF target. While all cores are enabled on the target for NVMe-oF, SPDK runs on 1, 2, and 3 cores.

of operations-per-second) for small objects (800B) when using SPDK NVMe-oF target. DAS and NVMe-oF performance are the same as in Figure 9 and are shown in the figure for comparison. The performance difference between all configurations is marginal, but using SPDK further reduces the load on the target server. Unlike NVMe-oF, SPDK is able to match RocksDB local performance with only a single core, reducing overall system utilization on the target from $12 \%$ for NVMe-oF to a mere $1 \%$ for SPDK.

\section{RELATED WORK}

Disaggregation is a well-known approach to achieve computing hardware modularity and to address imbalanced resource requirements that results in overprovisioning [17]. HDD disaggregation $[1,22,34,41,51,62]$ is common, because network overheads are insignificant compared to the latency of accessing data on a spinning disk. This article focuses on PCIe-based SSD disaggregation, which poses significant challenges due to the raw throughput and latency of NVMe-SSD. 
Klimovic et al. [31] evaluate Flash disaggregation with iSCSI. They show that even though iSCSI remote access introduces a $20 \%$ throughput drop at the application level, disaggregation makes up for that overhead by independently scaling CPU and Flash resources. We extend their work by evaluating disaggregation over NVMe-oF and show that NVMe-oF reduces the application performance impact to a bare minimum, thus greatly improving cost efficiency and practicality.

Hyper-Convergence Infrastructure (HCI) is a software-defined approach that bundles commodity servers, each containing both compute and storage, into a clustered pool [53]. It abstracts the underlining hardware through a virtual computing platform, and allows management and scaling of compute and storage through a centralized management interface. $\mathrm{HCI}$ simplifies the infrastructure management and is gaining popularity within the enterprise segment $[50,53]$. In this article, we focus on web-scale data centers, which are generally not hyperconverged $[18,55]$. Specifically, to better utilize hardware resources, web-scale data centers use few types of (sometimes highly customized) servers, each with a different balance of compute, storage, memory, and network resources (e.g., [22]). Flash disaggregation fits well within this deployment model, as some of the server classes are already tuned specifically for storage. Moreover, NVMe-oF, HCI, and storage disaggregation are not mutually exclusive: Excelero's utilizes NVMe-oF for its NVMesh architecture that supports both hyperconverged and disaggregated storage configurations [13]. Likewise, hybrid HCI and disaggregation architectures are also being explored [12, 58].

Since NVMe-oF v1.0 Specification [48] is relatively new, there is limited literature and analysis available. The design and architecture can be found in $[6,29,44]$, but none provide detailed performance analysis. Xu et al. [65] provide an in-depth analysis of NVMe drive and their performance benefits for multiple cloud databases. Our work shows how NVMe's benefits can be extended over the network using NVMe-oF.

Several works propose I/O stack optimizations to take advantage of fast NVMe devices [5, 59, 67]. Recently, Intel introduced SPDK [24]: a set of tools and libraries for writing high-performance user-mode storage applications that reduce kernel context switches and eliminate interrupt handling overheads. Moving drivers into userspace and operating in a polled mode will improve CPU utilization for both NVMe-oF and iSCSI, as our initial studies have shown. The SPDK toolkit also contains an NVMe-oF target application [25] that runs the entire NVMe-oF target path in userspace, bypassing the kernel. While the use of SPDK on the host requires either a specialized file system $[11,28,60]$ or application modifications (because applications now need to explicitly manage the drives), using SPDK NVMe-oF target on the storage node is completely transparent to the host's applications. This article focuses on disaggregation through minimal changes to the host; we leave detailed evaluation of the full SPDK NVMe-oF path (i.e., host-to-target-to-host) to future work.

NVMe-oF requires an RDMA-capable network. With both RoCE and iWARP enabling RDMA over Ethernet (or IP), RDMA is becoming more popular in the data center domain, and more applications are being ported to utilize it. Some notable implementation examples in addition to NVMe-oF include SRP (SCSI RDMA Protocol) [61], iSER (iSCSI Extensions for RDMA) [32], Windows SMB Direct [42], Luster [8], Hadoop [37], Crail [21], and Ceph [30].

\section{CONCLUSION}

Data center applications' requirements for high-performance storage keep increasing. Consequently, NVMe-SSDs are increasingly being deployed to cater to the ever-growing demand for throughout and low-latency access. As NVMe-SSDs are becoming more prevalent in the data center, Flash disaggregation has been proposed to improve resource efficiency and reduce the overall cost of NVMe deployment. However, inefficiencies of existing remote storage protocols have hindered performance and limited disaggregation benefits. This work revisits Flash disaggregation 
using NVMe-over-Fabrics: a new protocol designed specifically to extend the high performance of NVMe to remote servers.

We provide the first, in-depth analysis of NVMe-oF, characterizing end-to-end performance as well as the resource overheads on both the host and the target. Using synthetic stress-tests and two use cases from the data center domain (RocksDB and MySQL), we compare NVMe-oF with direct attached storage and with disaggregation via iSCSI. We show that NVMe-oF delivers high performance and low latencies with minimal CPU overheads, consistent with its design goals. Our results indicate that for the most part, application will not see noticeable difference between accessing local storage vs. remote storage over NVMe-oF. Lastly, we show that iSCSI introduces significant latencies for NVMe disaggregation (10× in some cases) and can degrade application performance by up to $40 \%$.

\section{REFERENCES}

[1] Amazon. 2008. Amazon Elastic Block Store. Retrieved from https://aws.amazon.com/ebs/.

[2] Jens Axboe. 2014. FIO. Retrieved from https://github.com/axboe/fio.

[3] Luiz André Barroso, Jimmy Clidaras, and Urs Hölzle. 2013. The datacenter as a computer: An introduction to the design of warehouse-scale machines. Synth. Lect. Comput. Architect. 8, 3 (2013), 1-154.

[4] X bit labs. 2016. OCZ Demos $4 \mathrm{TiB}, 16 \mathrm{TiB}$ Solid-State Drives for Enterprise. https://web.archive.org/web/ 20130325121004/http:/www.xbitlabs.com/news/storage/display/20120110180208_OCZ_Demos_4TB_16TB_Solid_ State_Drives_for_Enterprise.html.

[5] Matias Bjørling, Jens Axboe, David Nellans, and Philippe Bonnet. 2013. Linux block IO: Introducing multi-queue SSD access on multi-core systems. In Proceedings of the 6th International Systems and Storage Conference (SYSTOR'13). ACM, New York, NY, Article 22. DOI : https://doi.org/10.1145/2485732.2485740

[6] Brandon Hoff. 2016. RDMA Interconnects Paving the Way for NVMe over Fabrics Technology. Retrieved from http://www.roceinitiative.org/.

[7] David Cohen, Thomas Talpey, Arkady Kanevsky, Uri Cummings, Michael Krause, Renato Recio, Diego Crupnicoff, Lloyd Dickman, and Paul Grun. 2009. Remote direct memory access over the converged enhanced ethernet fabric: Evaluating the options. In Proceedings of the 17th IEEE Symposium on High Performance Interconnects (HOTI'09). IEEE, 123-130.

[8] Chelsio Communications. 2014. Luster over iWARP RDMA at 40Gbps. http://www.chelsio.com/wp-content/uploads/ resources/Lustre-Over-iWARP-vs-IB-FDR.pdf.

[9] RDMA Consortium. 2002. Architectural Specifications for RDMA over TCP/IP. Technical Report. Retrieved from https:// www.rdmaconsortium.org/.

[10] Transaction Processing Performance Council. 2010. TPC-C Benchmark Standard Specification, Revision 5.11. Retrieved from http://www.tpc.org/tpc_documents_current_versions/pdf/tpc-c_v5.11.0.pdf.

[11] Brendan Cully, Jake Wires, Dutch Meyer, Kevin Jamieson, Keir Fraser, Tim Deegan, Daniel Stodden, Geoffre Lefebvre, Daniel Ferstay, and Andrew Warfield. 2014. Strata: High-performance scalable storage on virtualized non-volatile memory. In Proceedings of the 12th USENIX Conference on File and Storage Technologies (FAST'14). 17-31.

[12] Datium. 2018. Open Convergence. Retrieved from http://www.datrium.com/open-convergence/.

[13] Excelero. 2017. Excelero NVMesh. Retrieved from https://www.excelero.com/product/nvmesh/.

[14] Facebook. 2018. RocksDB users. Retrieved from https://github.com/facebook/rocksdb/blob/master/USERS.md.

[15] Facebook Inc. 2015. RocksDB: A persistent key-value store for fast storage environments. Retrieved from http:// rocksdb.org.

[16] FusionIO. 2013. Fusion-io flash memory as RAM relief.

[17] Sangjin Han, Norbert Egi, Aurojit Panda, Sylvia Ratnasamy, Guangyu Shi, and Scott Shenker. 2013. Network support for resource disaggregation in next-generation datacenters. In Proceedings of the 12th ACM Workshop on Hot Topics in Networks. ACM, 10.

[18] Kieran Harty. 2016. Don't Confuse Hyperconvergence With Web-Scale. Retrieved from http://www. networkcomputing.com/data-centers/dont-confuse-hyperconvergence-web-scale/445839104.

[19] HGST. 2014. LinkedIn scales to 200 million users with PCIe Flash storage from HGST. Retrieved from https://www. hgst.com/sites/default/files/resources/LinkedIn-Scales-to-200M-Users.pdf.

[20] Amber Huffman. 2012. NVM Express Revision 1.1. Retrieved from http://www.nvmexpress.org/.

[21] IBM Research. 2017. Crail. Retrieved from http://www.crail.io/.

[22] Facebook Inc. 2015. Open Compute Project. Retrieved from http://www.opencompute.org/projects. 
[23] Intel. 2016. Intel Xeon Processor E5-2699 v4. Retrieved from https://ark.intel.com/products/91317/Intel-XeonProcessor-E5-2699-v4-55M-Cache-2_20-GHz.

[24] Intel. 2016. Storage Performance Development Kit. Retrieved from http://www.spdk.io/.

[25] Intel. 2017. SPDK NVMe over Fabrics Target. Retrieved from http://www.spdk.io/doc/nvmf.html.

[26] Intel. 2017. SPDK NVMe over Fabrics Target Programming Guide. Retrieved from http://www.spdk.io/doc/nvmf_tgt_ pg.html.

[27] Svilen Kanev, Juan Pablo Darago, Kim Hazelwood, Parthasarathy Ranganathan, Tipp Moseley, Gu-Yeon Wei, and David Brooks. 2015. Profiling a warehouse-scale computer. In Proceedings of the 42nd Annual International Symposium on Computer Architecture (ISCA'15). ACM, New York, NY, 158-169. DOI : https://doi.org/10.1145/2749469.2750392

[28] Hyeong-Jun Kim, Young-Sik Lee, and Jin-Soo Kim. 2016. NVMeDirect: A user-space I/O framework for applicationspecific optimization on NVMe SSDs. In Proceedings of the 8th USENIX Workshop on Hot Topics in Storage and File Systems (HotStorage'16). USENIX Association, Denver, CO. Retrieved from https://www.usenix.org/conference/ hotstorage16/workshop-program/presentation/kim.

[29] John Kim and David Fair. 2016. How ethernet RDMA protocols iWARP and RoCE support NVMe over fabrics (Ethernet Storage Forum). SNIA. Retrieved from https://www.snia.org/sites/default/files/ESF/How_Ethernet_RDMA_ Protocols_Support_NVMe_over_Fabrics_Final.pdf.

[30] John F. Kim. 2014. Accelerating Ceph with flash and high speed networks. In Proceedings of the Storage Developer Conference. SNIA.

[31] Ana Klimovic, Christos Kozyrakis, Eno Thereska, Binu John, and Sanjeev Kumar. 2016. Flash storage disaggregation. In Proceedings of the Eleventh European Conference on Computer Systems (EuroSys'16). ACM, New York, NY, Article 29, 15 pages. DOI : https://doi.org/10.1145/2901318.2901337

[32] Mike Ko, J. Hufferd, M. Chadalapaka, Uri Elzur, H. Shah, and P. Thaler. 2003. iSCSI extensions for RDMA specification (version 1.0). Release Specification of the RDMA Consortium (2003).

[33] Percona Lab. 2008. tpcc-mysql. Retrieved from https://github.com/Percona-Lab/tpcc-mysql.

[34] Edward K. Lee and Chandramohan A. Thekkath. 1996. Petal: Distributed virtual disks. SIGOPS Oper. Syst. Rev. 30, 5 (Sept. 1996), 84-92. DOI : https://doi.org/10.1145/248208.237157

[35] Jiuxing Liu, Jiesheng Wu, Sushmitha P. Kini, Pete Wyckoff, and Dhabaleswar K. Panda. 2003. High performance RDMA-based MPI implementation over InfiniBand. In Proceedings of the 17th Annual International Conference on Supercomputing. ACM, 295-304.

[36] Charles Loboz. 2012. Cloud resource usage-Heavy tailed distributions invalidating traditional capacity planning models. F. Grid Comput. 10, 1 (Mar. 2012), 85-108. DOI : https://doi.org/10.1007/s10723-012-9211-x

[37] Xiaoyi Lu, Nusrat S. Islam, Md Wasi-Ur-Rahman, Jithin Jose, Hari Subramoni, Hao Wang, and Dhabaleswar K. Panda. 2013. High-performance design of Hadoop RPC with RDMA over InfiniBand. In Proceedings of the 42nd International Conference on Parallel Processing (ICPP'13). IEEE, 641-650.

[38] Charlie Manese. 2014. Facebook and open compute, designing for efficiency and scale. SC14 Energy Efficient High Performance Computing Working Group.

[39] Mellanox. 2015. Connect X-4 VPI 100Gb. Retrieved from http://www.mellanox.com/related-docs/prod_adapter_ cards/PB_ConnectX-4_VPI_OCP.pdf.

[40] Mellanox. 2015. SN2700. Retrieved from https://www.mellanox.com/related-docs/prod_eth_switches/PB_SN2700. pdf.

[41] James Mickens, Edmund B. Nightingale, Jeremy Elson, Krishna Nareddy, Darren Gehring, Bin Fan, Asim Kadav, Vijay Chidambaram, and Osama Khan. 2014. Blizzard: Fast, cloud-scale block storage for cloud-oblivious applications. In Proceedings of the 11th USENIX Conference on Networked Systems Design and Implementation (NSDI'14). USENIX Association, Berkeley, CA, 257-273. DOI : http://dl.acm.org/citation.cfm?id=2616448.2616473

[42] Microsoft. 2016. Improve Performance of a File Server with SMB Direct. Retrieved from https://technet.microsoft. com/en-us/library/jj134210(v=ws.11).aspx.

[43] Dave Minturn. 2016. NVMe over fabrics linux driver eco system. In Proceedings of the NVMe All Hands Meeting.

[44] Dave Minturn and J. Metz. 2015. Under the hood with NVMe over fabrics. In Proceedings of the Ethernet Storage Forum. SNIA. Retrieved from http://www.snia.org/sites/default/files/ESF/NVMe_Under_Hood_12_15_Final2.pdf.

[45] MySQL. 1998. MySQL. Retrieved from https://www.mysql.com/.

[46] MySQL. 2018. MySQL Customers. Retrieved from https://www.mysql.com/customers.

[47] Dell Networking. 2015. RDMA over converged ethernet technical brief. http://pleiades.ucsc.edu/doc/dell/network/ Dell_Networking_RoCE_Configuration.pdf.

[48] NVM Express. 2016. NVM Express over Fabric 1.0. Retrieved from http://www.nvmexpress.org/.

[49] Jian Ouyang, Shiding Lin, Song Jiang, Zhenyu Hou, Yong Wang, and Yuanzheng Wang. 2014. SDF: Softwaredefined flash for web-scale internet storage systems. In Proceedings of the 19th International Conference on 
Architectural Support for Programming Languages and Operating Systems (ASPLOS'14). ACM, New York, NY, 471484. DOI : https://doi.org/10.1145/2541940.2541959

[50] Julia Parlmer and Stanley Zaffos. 2016. How to Determine When Hyperconverged Integrated Systems Can Replace Traditional Storage. Technical Report. Gartner.

[51] Kestutis Patiejunas and Amisha Jaiswal. 2016. Facebook's disaggregated storage and compute for Map/Reduce. In Proceedings of Data @Scale.

[52] Ro Recio, P. Culley, D. Garcia, J. Hilland, and B. Metzler. 2005. An RDMA Protocol Specification. Technical Report. IETF Internet-draft draft-ietf-rddp-rdmap-03.txt (work in progress).

[53] Simon Robinson, John Abott, and Tim Stammers. 2015. The Emergence of Hyperconvergence. Technical Report. 451 Research.

[54] Steven Rostedt. 2008. ftrace-Function Tracer. Retrieved from https://www.kernel.org/doc/Documentation/trace/ ftrace.txt.

[55] Brandon Salmon. 2015. Web scale vs. hyperconverged: Understand the differences. Retrieved from http://www. infoworld.com/article/3005572/enterprise-architecture/web-scale-vs-hyperconverged-understand-the-differences. html.

[56] Samsung. 2015. PM1725 NVMe PCIe SSD. Retrieved from http://www.samsung.com/semiconductor/global/file/ insight/2015/11/pm1725-ProdOverview-2015-0.pdf.

[57] Bianca Schroeder, Raghav Lagisetty, and Arif Merchant. 2016. Flash reliability in production: The expected and the unexpected. In 14th USENIX Conference on File and Storage Technologies (FAST'16). USENIX Association, Santa Clara, CA, 67-80. Retrieved from http://usenix.org/conference/fast16/technical-sessions/presentation/schroeder.

[58] Simon Sharwood. 2016. Disaggregated hyper-convergence thinks storage outside the box. Retrieved from https:// www.theregister.co.uk/2016/03/24/disaggregated_hyper_convergence/.

[59] Woong Shin, Qichen Chen, Myoungwon Oh, Hyeonsang Eom, and Heon Y. Yeom. 2014. OS I/O path optimizations for flash solid-state drives. In Proceedings of the USENIX Annual Technical Conference (USENIX-ATC'14). USENIX Association, Philadelphia, PA, 483-488. Retrieved from https://www.usenix.org/conference/atc14/technical-sessions/ presentation/shin.

[60] Y. Son, N. Y. Song, H. Han, H. Eom, and H. Y. Yeom. 2014. A user-level file system for fast storage devices. In 2014 International Conference on Cloud and Autonomic Computing. 258-264. DOI : https://doi.org/10.1109/ICCAC.2014.14

[61] T10. 2003. SCSI RDMA protocol-2 (SRP-2). Retrieved from http://www.t10.org/ftp/t10/drafts/srp2/srp2r00a.pdf.

[62] VMWare. 2017. VMware vSAN. Retrieved from http://www.vmware.com/products/virtual-san.html.

[63] Benjamin Walker. 2016. SPDK: Building blocks for scalable, high performance storage applications (Storage Developer Conference). SNIA. Retrieved from http://www.snia.org/sites/default/files/SDC/2016/presentations/performance/ BenjaminWalker_SPDK_Building_Blocks_SDC_2016.pdf.

[64] Andrew Warfield. 2013. Architecting for "problematically fast" flash. COHO Data Virtualization Field Day 3. https: //techfieldday.com/event/sfd4/.

[65] Qiumin Xu, Huzefa Siyamwala, Mrinmoy Ghosh, Tameesh Suri, Manu Awasthi, Zvika Guz, Anahita Shayesteh, and Vijay Balakrishnan. 2015. Performance analysis of NVMe SSDs and their implication on real world databases. In Proceedings of the 8th ACM International Systems and Storage Conference (SYSTOR'15). ACM, New York, NY, Article 6, 11 pages. DOI : https://doi.org/10.1145/2757667.2757684

[66] Jisoo Yang, Dave B. Minturn, and Frank Hady. 2012. When poll is better than interrupt. In Proceedings of the 10th USENIX Conference on File and Storage Technologies (FAST'12). USENIX Association, Berkeley, CA, 3-3. DOI : http:// dl.acm.org/citation.cfm?id=2208461.2208464

[67] Young Jin Yu, Dong In Shin, Woong Shin, Nae Young Song, Jae Woo Choi, Hyeong Seog Kim, Hyeonsang Eom, and Heon Young Yeom. 2014. Optimizing the block I/O subsystem for fast storage devices. ACM Trans. Comput. Syst. 32, 2, Article 6 (June 2014). DOI : https://doi.org/10.1145/2619092

Received April 2018; revised July 2018; accepted July 2018 